\title{
Remarks on the Modular Operator and Local Observables
}

\author{
Carlo Rigotti* \\ Istituto Matematico "G. Castelnuovo", Università di Roma, I-00100 Roma, Italy
}

\begin{abstract}
In this paper we give a characterization of the modular group of a von Neumann algebra $\mathscr{R}$, with a cyclic and separating vector, which provides at the same time a necessary and sufficient condition so that two von Neumann algebras $\mathscr{R}_{1}$ and $\mathscr{R}_{2}$, such that $\mathscr{R}_{1} \subseteq \mathscr{R}_{2}^{\prime}$, are the mutual commutants, i.e. $\mathscr{R}_{1}=\mathscr{R}_{2}^{\prime}$.

An application is made to the duality property in Quantum Field Theory, and we give a sufficient condition for PCT invariance in a theory of local observables.
\end{abstract}

\section{Introduction}

It is known that if $\mathscr{R}$ is a von Neumann algebra with a cyclic and separating vector $\Omega$, then the associated modular operator is characterized by the following conditions :

i) $\Delta=\Delta^{*}, \Delta>0$;

ii) for each $t \in \mathbb{R} \Delta^{i t} \Omega=\Omega$;

iii) for each $t \in \mathbb{R} \Delta^{i t} \mathscr{R} \Delta^{-i t}=\mathscr{R}$;

iv) the automorphism group $\sigma_{t}=\Delta^{i t} \cdot \Delta^{-i t}$, satisfies the KMS condition for the state $\omega_{0}=(\Omega, \Omega)$.

Recall that $\Delta^{1 / 2}$ is the modulus in the polar decomposition of the *-operator $A \Omega \rightarrow A * \Omega, A \in \mathscr{R}$; the phase $J$ is an antiunitary involution such that $J \Delta^{1 / 2} A \Omega=A * \Omega$, and $J \mathscr{R} J=\mathscr{R}^{\prime}$. By these relations $\Delta^{1 / 2} \mathscr{R}^{\mathrm{sa}} \Omega=\mathscr{R}^{\prime \mathrm{sa}} \Omega$, where we denote with $\mathscr{R}^{\text {sa }}$ the selfadjoint operators of $\mathscr{R}[8]$.

Conversely the KMS condition is easily implied by the condition

iv') $\Delta^{1 / 2} \mathscr{R}^{\mathrm{sa}} \Omega \subset \mathscr{R}^{\prime \mathrm{sa}} \Omega$.

In this note we show that condition iv') independently from Tomita-Takesaki theory, implies a commutation theorem, and at the same time characterizes the modular group, producing another proof of the uniqueness of the modular automorphisms.

* Partially supported by C.N.R. 
This is suggested by the proof of duality in field theory [1], and in analogy with that analysis can possibly be further generalized to *-algebras of unbounded operators.

\section{2.}

In this paper the Rieffel-van Daele commutation theorem is very important [6]; we will use the following simplified form of the theorem:

Theorem 1. Let $\mathscr{R}_{1}$ and $\mathscr{R}_{2}$ be von Neumann algebras on the Hilbert space $\mathscr{H}$, with a cyclic and separating vector $\Omega$. We assume that $\mathscr{R}_{1}$ and $\mathscr{R}_{2}$ commute. Then $\mathscr{R}_{1}=\mathscr{R}_{2}^{\prime}$ if and only if the following condition is satisfied: if $\xi \in \mathscr{H}$ and for each $A \in \mathscr{R}_{1}^{\text {sa }}, B \in \mathscr{R}_{2}^{\text {sa }}$ we have

$$
\operatorname{Im}(A \Omega, \xi)=0, \quad \operatorname{Re}(B \Omega, \xi)=0
$$

then $\xi=0$.

We shall utilize the following lemma.

Lemma 2. Let $\mathscr{R}$ be a von Neumann algebra with a cyclic and separating vector $\Omega$, and let $U(t)$ be an unitary strongly continuous group such that $U(t) \Omega=\Omega$, and $U(t) \mathscr{R} U(t)^{*}=\mathscr{R}$ for every $t \in \mathbb{R}$.

If we assume that the unbounded operator $U(i)=e^{-K}$ (where $K$ is the generator of the group $U(t)$ ) satisfies the following condition

$$
\mathscr{R} \Omega \subseteq \mathscr{D}(U(i)) \text { and } U(i) \mathscr{R}^{\mathrm{sa}} \Omega \subseteq \mathscr{R}^{\mathrm{sa}} \Omega
$$

then $U(t)=I$ for each $t \in \mathbb{R}$.

Proof. Let $A \in \mathscr{R}^{\text {sa }}$ and $B \in \mathscr{R}^{\mathrm{sa}}$. Since $B \Omega \in \mathscr{D}(U(i)) \subseteq \mathscr{D}(U(z))$, for $z \in \mathbb{C}$ and $\operatorname{Im} z \in[0,1]$ we can define the function

$$
f(z)=(A \Omega, U(z) B \Omega)
$$

by the spectral theory of selfadjoint operators $f$ is analytic for $\operatorname{Im} z \in(0,1)$ and continuous for $\operatorname{Im} z \in[0,1]$. On the real axis $f$ is a real function, because $A U(t) B U(z)^{*}$ is selfadjoint; on the axis $\operatorname{Im} z=1$ we have

$$
f(t+i)=(A \Omega, U(t) U(i) B \Omega) ;
$$

but there is $\hat{B} \in \mathscr{R}^{\text {sa }}$ such that $U(i) B \Omega=\hat{B} \Omega$, hence $f(t+i)$ is real. By [10], Lemma 1.6, $f$ is bounded on the strip. By the principle of analytic reflection we can extend $f$ to an entire bounded function, and by Liouville theorem $f$ is constant. We have proved

$$
\forall t \in \mathbb{R}(A \Omega, U(t) B \Omega)=(A \Omega, B \Omega)
$$

but $\Omega$ is cyclic and separating for $\mathscr{R}$, and we obtain $U(t) \equiv I$.

The following lemma is well-known [8]:

Lemma 3. Let $U$ be an unitary operator such that $U \Omega=\Omega$ and $U \mathscr{R} U^{*}=\mathscr{R}$, where $\Omega$ is a cyclic and separating vector for the von Neumann algebra $\mathscr{R}$. Then $U$ commutes with the modular group associated to $\Omega$. 


\section{3.}

The following gives a density condition, and at the same time a characterization of the modular group:

Theorem 4. Let $R_{1}$ and $R_{2}$ be von Neumann algebras with a common cyclic vector $\Omega$. We assume that $R_{1}$ and $R_{2}$ commute, and there is a strongly continuous unitary group $U(t)$ satisfying the following conditions:

i) $U(t) \mathscr{R}_{l} U(t)^{*}=\mathscr{R}_{l}$ for $\quad l=1,2, t \in \mathbb{R}$;

ii) $U(t) \Omega=\Omega, \quad t \in \mathbb{R}$;

iii) $\mathscr{R}_{1} \Omega \cong(U(i)), \quad U(i) \mathscr{R}_{1}^{\mathrm{sa}} \Omega \cong \mathscr{R}_{2}^{\mathrm{sa}}$.

Then $\mathscr{R}_{1}=\mathscr{R}_{2}^{\prime}$ and $\Delta^{-i t / 2}=U(t)$.

Proof. We utilize the Rieffel-Van Daele commutation theorem. Let $\xi \in \mathscr{H}$, and suppose that for $A \in \mathscr{R}_{1}^{\text {sa }}$ and $B \in \mathscr{R}_{2}^{\text {sa }},(\xi, A \Omega)$ is real, and $(\xi, B \Omega)$ is pure imaginary. We want to show that $\xi=0$.

We define on the strip $\bar{I}=\{z \in \mathbb{C} \mid \operatorname{Im} z \in(0,1)\}^{-}$the function

$$
f(z)=(\xi, U(z) A \Omega) \text {. }
$$

By [10], Lemma 1.6, $f$ is analytic on $I$, continuous and bounded on $\bar{I}$. Moreover $f(t)=(\xi, U(t) A \Omega)=\left(\xi, U(t) A U(t)^{*} \Omega\right)$ by condition ii, hence $f$ is real on the real axis by condition i.

On the axis $\operatorname{Im} z=1$, we have $f(t+i)=(\xi, U(t) U(i) A \Omega)$, but by condition iii) there is $\hat{A} \in \mathscr{R}_{2}^{\text {sa }}$ such that $U(i) A \Omega=\hat{A} \Omega$. Hence $f(t+i)=(\xi, U(t) \hat{A} \Omega)$ is pure imaginary because $U(t) \hat{A} U(t)^{*} \in \mathscr{R}_{2}^{\text {sa }}$.

Since $f$ is real on the real axis and pure imaginary on the axis $\operatorname{Im} z=1$, by the principle of analytic reflection, we can extend it to an entire function. By Liouville Theorem $f$ is constant; but $f$ is real on the real axis, whereas it is purely imaginary on the axis $\operatorname{Im} z=1$, hence $f \equiv 0$.

In particular for every $A \in \mathscr{R}_{1}^{\text {sa }}$ we have $(\xi, A \Omega)=0$; then $\xi \in(R, \Omega)^{\perp}$ and we obtain $\xi=0$. By Theorem $1 \mathscr{R}_{1}=\mathscr{R}_{2}^{\prime}$. We know, by Lemma 3 , that $\Delta^{i s}$ and $U(t)$ commute for every $t, s \in \mathbb{R}$. Trivially the unitary group $V(t)=\Delta^{i t / 2} U(t)$ is a strongly continuous unitary group satisfying the following conditions:

i) $V(t) \Omega=\Omega \quad t \in \mathbb{R}$;

ii) $V(t) \mathscr{R}_{1} V(t)^{*}=\mathscr{R}_{1} \quad t \in \mathbb{R}$;

iii) $\mathscr{R}_{1} \Omega \subseteq \mathscr{D}(V(i)), \quad V(i) \mathscr{R}_{1}^{\mathrm{sa}} \Omega \cong \mathscr{R}_{1}^{\mathrm{sa}} \Omega$.

Hence $V(t) \equiv I$ by the Lemma 2 , and $U(t)=\Delta^{-i t / 2}$.

Remark. Note that the implication $\mathscr{R}_{1}=\mathscr{R}_{2}^{\prime}$ in the theorem is independent from Tomita-Takesaki theory. If $\mathscr{R}_{1}$ is a *algebra (not a von Neumann algebra) fulfilling the assumption of Theorem 4 , then we can conclude $\mathscr{R}_{1}^{\prime \prime}=\mathscr{R}_{2}^{\prime}$, by the Rieffel-Van Daele commutation theorem [6].

4.

We want to apply the previous result to proof of the duality for the von Neumann algebras $\mathscr{R}\left(W_{R}\right)$ and $\mathscr{R}\left(W_{L}\right)$ associated with a hermitian scalar field $\varphi$, satisfying the 
Bisognano-Wichmann condition [1]: for every real test functions $f, g \in \mathscr{S}\left(\mathbb{R}^{4}\right)$ such that supp $f$ and supp $g$ are space-like separated, the field operators $\varphi(f)$ and $\varphi(g)$ have selfadjoint closure whose spectral projectors commute.

Let $V(t)$ be the representation of the group of the velocity transformations whose action is described by the matrix

$$
\Lambda(t)=\left(\begin{array}{cccc}
\operatorname{ch} t & \operatorname{sh} t & 0 & 0 \\
\operatorname{sh} t & \operatorname{ch} t & 0 & 0 \\
0 & 0 & 1 & 0 \\
0 & 0 & 0 & 1
\end{array}\right)
$$

Furthermore define $J=\vartheta U\left(R_{e_{1}}(\pi)\right)$, where $\vartheta$ is the PCT conjugation, and $R_{e_{1}}(\pi)$ is the rotation of $\pi$ about the $x_{1}$ axis.

If $W_{R}=\left\{\chi \in \mathbb{R}^{4}\left|\chi_{1}>\right| \chi_{0} \mid\right\}=-W_{L}$, we denote $\mathscr{R}\left(W_{k}\right)$ (for $k=L, R$ ) the von Neumann algebra generated by the spectral projector of the operators $\overline{\varphi(f)}$, where $f$ is a real test function of $\mathscr{S}\left(\mathbb{R}^{4}\right)$ whose support is contained in $W_{k}$. By the condition of Bisognano and Wichmann $\mathscr{R}\left(W_{R}\right) \subseteq \mathscr{R}\left(W_{L}\right)^{\prime}$; in [1] they prove that for each $A \in \mathscr{R}\left(W_{R}\right)$

$$
A \Omega \in \mathscr{D}(V(i \pi)), \quad V(i \pi) A \Omega=J A^{*} \Omega .
$$

By the action of the PCT operator $\vartheta$ on the field we have $J \Omega=\Omega$ and $J \mathscr{R}\left(W_{R}\right) J$ $=\mathscr{R}\left(W_{L}\right)$; hence by Theorem $4 \mathscr{R}\left(W_{R}\right)=\mathscr{R}\left(W_{L}\right)^{\prime}$. Trivially the operator $J V(i \pi)$ is the $S$ operator of the Tomita-Takesaki theory (note that $\mathscr{R}\left(W_{R}\right) \Omega$ is a core for $V(i \pi)$, since it is invariant for the group $\{V(t)\})$. If $\mathscr{A}(\mathcal{O})$ is the local net generated by the field operators, we have for a double cone $\mathcal{O}$ contained in $W_{R}$

$$
\mathscr{R}\left(W_{R}\right)=\left\{\bigcup_{t \in \mathbb{R}} V(t) \mathscr{A}(\mathcal{O}) V(t)^{*}\right\}^{\prime \prime}=\left\{\bigcup_{\mathcal{O} \subseteq W_{R}} \mathscr{A}(\mathcal{O})\right\}^{\prime \prime}
$$

because these von Neumann algebras have the same $S$ operator [8]; we also obtain

$$
\text { (*) } \mathscr{A}\left(\mathcal{O}^{\prime}\right)^{-W}=\left\{\bigcup_{\Lambda W_{R} \subseteq \mathcal{O}^{\prime}} U(\Lambda) \mathscr{R}\left(\mathrm{W}_{R}\right) U(\Lambda)^{*}\right\}^{\prime \prime},
$$

where $\mathscr{A}\left(\mathcal{O}^{\prime}\right)$ is the $C^{*}$-algebra generated by the von Neumann algebras $\mathscr{A}\left(\mathcal{O}_{\alpha}\right)$ with $\mathcal{O}_{\alpha}$ space-like separated with $\mathcal{O}$.

Let $\mathscr{A}$ be a net of local algebras [4]. One may define a dual net by setting $\mathscr{A}^{d}(\mathcal{O})=\mathscr{A}\left(\mathcal{O}^{\prime}\right)^{\prime}[7]$. The net $\mathscr{A}^{d}$ satisfies the properties :

i) $\mathscr{A} \subseteq \mathscr{A}^{d}$.

ii) $\mathscr{A}$ is local [i.e. $\mathscr{A}\left(\mathcal{O}_{1}\right)$ commute with $\mathscr{A}\left(\mathcal{O}_{2}\right)$ if $\mathscr{O}_{1}$ and $\mathscr{O}_{2}$ are space-like separated] iff $\mathscr{A} \subseteq \mathscr{A}^{d}$.

iii) $\mathscr{A}$ satisfies the duality iff $\mathscr{A}=\mathscr{A}^{d}$.

iv) If $\mathscr{A}_{1}$ and $\mathscr{A}_{2}$ are two net of local algebras, and $\mathscr{A}_{1} \subseteq \mathscr{A}_{2}$ then $\mathscr{A}_{2}^{d} \subseteq \mathscr{A}_{1}^{d}$.

We say that a net of local algebras satisfies assential duality if $\mathscr{A}^{d}=\mathscr{A}^{\text {dd }}$. Now if $\mathscr{A}$ is a local net, i.e. if $\mathscr{A} \subseteq \mathscr{A}^{d}$, then essential duality is fulfilled if and only if $\mathscr{A}^{d}$ is a local net [7].

In the Bisognano-Wichmann analysis, the condition $(*)$ implies that $\mathscr{A}^{d}$ is a local net; hence the local net generated by a hermitian scalar field satisfies essential duality. 
The above discussion can be generalized to a theory of local observables. Let $\mathscr{A}$ be a local net of local algebras Poincaré covariant, i.e. there is an unitary strongly continuous representation $\mathscr{U}$ of the Poincaré group $\mathscr{P}$, such that

$$
\forall\{\Lambda, a\} \in \mathscr{P} \quad U(\Lambda, a) \mathscr{A}(\mathcal{O}) U(\Lambda, a)^{*}=\mathscr{A}(\{\Lambda, a\} \mathcal{O})
$$

for every double cone $\mathcal{O}$. Let $\Omega$ be the vacuum (it is Poincaré invariant). The following theorem give a sufficient condition so that the local net $\mathscr{A}$ satisfies essential duality, independently from the existence of a Wightman field generating the net, and from the PCT invariance of the theory.

Theorem 5. Let $\mathscr{A}$ be a local net of local algebras Poincaré covariant, $\Omega$ the vacuum state. We call $\mathscr{R}\left(W_{R}\right)$ and $\mathscr{R}\left(W_{L}\right)$ the von Neumann algebras generated by the algebras $\mathscr{A}(\mathcal{O})$ with $\mathcal{O} \subseteq W_{R}\left(\mathcal{O} \subseteq W_{L}\right.$ respectively). Then $\mathscr{A}$ fulfills essential duality if for each double cone $\mathcal{O} \subseteq W_{R}$

$$
(* *) \quad \mathscr{A}(\mathcal{O}) \Omega \subseteq \mathscr{D}(U(i \pi)), \quad U(i \pi) \mathscr{A}(\mathcal{O})^{\mathrm{sa}} \Omega \subseteq \mathscr{R}\left(W_{L}\right)^{\mathrm{sa}} \Omega,
$$

where $U(t)=U(\Lambda(t), 0)$ implements the pure Lorentz transformations along the $x_{1}$ axis.

Proof. By remark to Theorem 4 we have that $U(t)$ is the modular group of $\mathscr{R}\left(W_{R}\right)$ associated with the cyclic and separating vector $\Omega$ (note $\Omega$ is an analytic vector for the energy) [2]; moreover $\mathscr{R}\left(W_{R}\right)=\mathscr{R}\left(W_{L}\right)^{\prime}$. By an argument similar to BisognanoWichmann proof, this implies essential duality.

Note that in the Bisognano-Wichmann situation condition $(* *)$ takes the more specific form:

$$
\begin{array}{ll} 
& \text { for each double cone } \mathcal{O} \subseteq W_{R} \\
(* * *) \quad & \mathscr{A}(\mathcal{O}) \Omega \subseteq \mathscr{D}(U(i \pi)), \quad U(i \pi) \mathscr{A}(\mathcal{O})^{\mathrm{sa}} \Omega \subseteq \mathscr{A}\left(\mathcal{O}^{j}\right)^{\mathrm{sa}} \Omega \\
& \text { where } \mathcal{O}^{j}=\left\{\chi \in \mathbb{R}^{4} \mid\left(-\chi_{0},-\chi_{1}, \chi_{2}, \chi_{3}\right) \in \mathcal{O}\right\} .
\end{array}
$$

This more restrictive assumption implies in the general case that there is a PCT operator $\vartheta$ such that for every Poincaré transformation $\{\Lambda, a\}$ we have $\vartheta U(\Lambda, a) \vartheta$ $=U(\Lambda,-a)$.

Theorem 6. Let $\mathscr{A}$ be a local net of local algebras Poincaré covariant, $\Omega$ the vacuum. If condition $(* * *)$ is fulfilled the anti-unitary operator $\vartheta=U\left(R_{e_{1}}(\pi)\right) J$ ( $J$ is the modular conjugation associated to the von Neumann algebra $\mathscr{R}\left(W_{R}\right)$ for the cyclic and separating vector $\Omega ; R_{e_{1}}(\pi)$ is the rotation of $\pi$ about the axis determined by the unit vector $\left.e_{1}=(0,1,0,0)\right)$ satisfies the following conditions:

1. $\vartheta^{2}=I, \vartheta \Omega=\Omega$;

2. for each double cone $\mathcal{O} \vartheta \mathscr{A}(\mathcal{O}) \vartheta=\mathscr{A}(-\mathcal{O})$;

3. for each Poincaré transformation $\{\Lambda, a\}, \vartheta U(\Lambda, a)=U(\Lambda,-a) \vartheta$.

In particular $\vartheta$ is independent from the wedge region which we use to define it.

Proof. By an argument of analytical extension $J$ commutes with $U\left(R_{e_{1}}(\pi)\right)$ and we have 1 . We want to prove that for $a \in \mathbb{R}^{4} U(I,-a)=\vartheta U(I, a) \vartheta$. As $\{U(I, a)\}$ is a 
group, it suffices to prove the equality

$$
U\left(I, a^{j}\right)=J U(I, a) J
$$

where $a^{j}=\left(-a_{0},-a_{1}, a_{2}, a_{3}\right)$, for $a \in W_{R}$.

If $z \in \mathbb{C}$ we define

$$
\Lambda(z)=\left(\begin{array}{cccc}
\operatorname{ch} z & \operatorname{sh} z & 0 & 0 \\
\operatorname{sh} z & \operatorname{ch} z & 0 & 0 \\
0 & 0 & 1 & 0 \\
0 & 0 & 0 & 1
\end{array}\right)
$$

this is an extension of the pure Lorentz transformations along the $x_{1}$ axis, and $z \rightarrow \Lambda(z)$ is an analytic entire function. By a simple computation if $\operatorname{Im} z \in(0, \pi)$ and $a \in W_{R}, \Lambda(z) a \in \mathscr{I}=\mathbb{R}^{4}+i V_{+}$.

By spectrum condition [2] there is an extension $T(\zeta)$ of the translations group on the tube $\overline{\mathscr{I}}$. The function $\zeta \in \overline{\mathscr{I}} \rightarrow T(\zeta)$ is analytic on $\mathscr{I}$, strongly continuous on $\overline{\mathscr{I}}$, and for $x \in \mathbb{R}^{4}$ we have $T(x)=U(I, x)$.

If $a \in W_{R}$ then $a+W_{R} \subseteq W_{R}$; hence if $A \in \mathscr{R}\left(W_{R}\right), U(I, a) A U(I,-a) \in \mathscr{R}\left(W_{R}\right)$. In particular $A \Omega, U(I, a) A \Omega \in \mathscr{D}(U(i \pi)) \subseteq \mathscr{D}(U(z))$ for $\operatorname{Im} z \in[0, \pi]$.

If $a \in W_{R}$ we define on the strip $G=\{z \in \mathbb{C} \mid \operatorname{Im} z \in(0, \pi)\}$

$$
f(z)=U(z) U(I, a) A \Omega-T(\Lambda(z) a) U(z) A \Omega
$$

this function is well-defined, analytic on $G$ and continuous on $\bar{G}$. One easily see that for every $t \in \mathbb{R} f(t)=0$, and by the principle of analytical continuation $f \equiv 0$. In particular $f(i \pi)=0$, i.e.

$$
U(i \pi) U(I, a) A \Omega=T(\Lambda(i \pi) a) U(i \pi) A \Omega ;
$$

but $\Lambda(i \pi) a=a^{j}$ and by Tomita-Takesaki theory we have

$$
J U(I, a) A^{*} \Omega=U\left(I, a^{j}\right) J A^{*} \Omega
$$

$\Omega$ is cyclic for $\mathscr{R}\left(W_{R}\right)$ and 4$)$ is proved. (Note that we have used condition (**) only).

If $\mathcal{O} \subseteq W_{R}$ is a double cone, then

$$
\vartheta \mathscr{A}(\mathcal{O}) \Omega \subseteq \mathscr{A}(-\mathcal{O}) \Omega
$$

because of the action of $U\left(R_{e_{1}}(\pi)\right)$ and condition $(* * *)$.

But $\vartheta \mathscr{R}\left(W_{R}\right) \vartheta=\mathscr{R}\left(-W_{R}\right)=\mathscr{R}\left(W_{L}\right)$, hence $\vartheta \mathscr{A}(\mathcal{O}) \vartheta \subseteq \mathscr{R}\left(W_{L}\right)$. Now if $A \in \mathscr{A}(\mathcal{O})$ $\vartheta A \vartheta \in \mathscr{R}\left(W_{L}\right)$, but $\vartheta A \vartheta \Omega=\vartheta A \Omega=A^{\prime} \Omega$ where $A^{\prime} \in \mathscr{A}(-\mathcal{O})$ and so $\vartheta A \vartheta \in \mathscr{A}(-\mathcal{O})$, as $\Omega$ is a separating vector for $\mathscr{R}\left(W_{L}\right)$. Hence $\vartheta \mathscr{A}(\mathcal{O}) \vartheta \subseteq \mathscr{A}(-\mathcal{O})$. If $\mathcal{O}$ is a double cone there is $a \in W_{R}$ such that $a+\mathcal{O} \subseteq W_{R}$; then

$$
\begin{aligned}
T(-a) \vartheta \mathscr{A}(\mathcal{O}) \vartheta T(a) & =\vartheta T(a) \mathscr{A}(\mathcal{O}) T(-a) \vartheta=\vartheta \mathscr{A}(\mathcal{O}+a) \vartheta \\
& \cong \mathscr{A}(-\mathcal{O}-a)=T(-a) \mathscr{A}(-\mathcal{O}) T(a) .
\end{aligned}
$$

This implies 2 .

We want to prove that for every $\Lambda \in L_{+}^{\uparrow} \vartheta$ and $U(\Lambda)$ commute. If $R$ is a rotation, the von Neumann algebra associated with the wedge region $R W_{R}$ is $\mathscr{R}\left(R W_{R}\right)$ $=U(R) \mathscr{R}\left(W_{R}\right) U(R)^{*}$, the modular group of $\mathscr{R}\left(R W_{R}\right)$ is $U_{R}(t)=U(R) U(t) U(R)^{*}$, and 
the modular conjugation is $J_{R}=U(R) J U(R)^{*}$. If $e$ is the unit vector $R e_{1}$ [where $\left.e_{1}=(0,1,0,0)\right]$, we denote $R_{e}(\pi)$ the rotation of $\pi$ about the axis determined by $e$. Trivially $R_{e}(\pi)=R R_{e_{1}}(\pi) R^{-1}$. If we denote $\vartheta_{R}=U\left(R_{e}(\pi)\right) J_{R}, \vartheta_{R}$ satisfies assumption 2. Since the local net is covariant $\vartheta_{R}$ commutes with $U_{R}(t)$.

Let $V_{R}=\vartheta_{R}$; then for each double cone $\mathcal{O} V_{R} \mathscr{A}(\mathcal{O}) V_{R}^{*}=\mathscr{A}(\mathcal{O})$ because $\vartheta$ and $\vartheta_{R}$ satisfy 2 ; moreover $V_{R} \Omega=\Omega$. By Lemma $3, V_{R}$ commutes with the modular group $U_{R}(t)$ (each wedge region is invariant for $V_{R}$ ). Because $\vartheta_{R}$ commutes with $U_{R}(t)$, we have proved that $\vartheta$ commutes with $U_{R}(t)$. Hence for every pure Lorentz transformation $\Lambda \vartheta$ commutes with $U(\Lambda)$.

The group generated by the pure Lorentz transformations is a normal subgroup of $L_{+}^{\uparrow}$, and $L_{+}^{\uparrow}$ has only trivial normal subgroup; so $L_{+}^{\uparrow}$ is the group generated by the pure Lorentz transformations, and $\vartheta$ commutes with $U\left(L_{+}^{\uparrow}\right)$.

Acknowledgements. I wish to thank Professor S. Doplicher for his help in the course of this work, and the continuous encouragements. I am also grateful to C. D'Antoni for interesting discussions on the applications of Rieffel-Van Daele commutation theorem to the proof of duality for quantum fields.

\section{References}

1. Bisognano,J., Wichmann, E.: On the duality condition for a Hermitian scalar field. J. Math. Phys. 16, 985 (1975)

2. Borchers, J.: On the vacuum state in quantum field theory. Commun. math. Phys. 1, 57 (1965)

3. Dixmier,J.: Les algebres d'operateurs dans l'espace hilbertien (les algebres de von Neumann). Paris : Gautier-Villars 1969

4. Doplicher,S., Haag, R., Roberts, J.: Local observables and particles statistics. I. Commun. math. Phys. 23, 199 (1971)

5. Reed,M., Simon,B.: Fourier analysis. Selfadjointness. New York-London: Academic Press 1975

6. Rieffel, M., Van Daele,A.: The commutation theorem for tensor products of von Neumann algebras. Bull. Lond. Math. Soc. 7, 257-260 (1975)

7. Roberts, J.: Local cohomology and its structural implications for field theory. Preprint (1977)

8. Takesaki, M.: Tomita theory of modular Hilbert algebras and its applications. Lecture notes in mathematics, Vol. 128. Berlin-Heidelberg-New York: Springer 1970

9. Wightman, A., Streater, R. : PCT, spin and statics and all that. New York: Benjamin 1964

10. Araki,H.: Positive cone, Radon Nicodym theorems, relative hamiltonian. Proceedings of the International School of Physics "Enrico Fermi", Varenna 1973 (Course LX)

11. Rieffel, M.: A commutation theorem and duality for free Bose fields. Commun. math. Phys. 39, $153-164$ (1974)

Communicated by R. Haag

Received December 20, 1977 
